\title{
Slit-lamp measurement of the anterior chamber depth
}

\author{
W A DOUTHWAITE AND D SPENCE \\ From the Undergraduate School of Studies in Optometry, University of Bradford
}

SUMmARY A technique is proposed which allows assessment of the anterior chamber depth with a standard table slit-lamp without any extra attachments. A final approximation is made which suggests that a $1^{\circ}$ change in the angle between the observation and illumination systems of the slit lamp when the end point is reached indicates a change in anterior chamber depth of $0.1 \mathrm{~mm}$.

The first recorded slit-lamp technique for measuring the depth of the anterior chamber was that used by Donders in 1872. In this technique the slit-lamp is focused on the corneal endothelium and then the anterior lens surface. The slit-lamp movement between these two positions gives the apparent depth of the anterior chamber. In 1914 Ulbrich utilised the Donders technique with the additional refinement of a micrometer drum to measure the slit-lamp movement accurately. The technique, of course, relies on the eye maintaining a fixed position during the time that the slit-lamp is moved from the endothelium to the anterior lens. This is a major drawback of the technique. Increased accuracy was claimed by Lindstedt,' who employed a spherical and a cylindrical lens to produce an astigmatic bundle of light rays. The spherical lens was focused on to the cornea and the cylindrical lens was moved until its power meridian focused on to the anterior lens. This allows a simultaneous comparison of the two images, and the lens positions are used to deduce the anterior chamber depth. The technique was further improved by Stenstrom ${ }^{2}$ with the addition of Scheiner discs to the optical system.

At present the commonest technique applied is that which uses parallel sided glass plates to displace half the image of an optical section until the corneal endothelium of one half coincides with the anterior lenticular surface of the other. Jaeger ${ }^{3}$ developed this approach, and it is employed in present-day pachometers. The pachometer has proved to be a rapid, convenient, and accurate method of estimating anterior chamber depth. But there is a need to have some means of recording the anterior chamber depth without recourse to expensive and sometimes in-

Correspondence to W A Douthwaite, $\mathrm{PhD}$, Undergraduate School of Studies in Optometry, University of Bradford, Bradford, West Yorkshire BD7 1DP. compatible accessories for the slit-lamp. Jacobs ${ }^{4}$ proposed a method of measuring the anterior chamber depth with a standard Haag-Streit 900 slitlamp. In this method the microscope is fixed at a $45^{\circ}$ angle to the illumination system. A slit is projected on to the patient's cornea, and the patient is instructed to fixate the slit. The slit is then focused on the anterior lens surface with its inferior edge concident with the centre of the pupil. The out-of-focus slit image on the cornea is then observed and its length adjusted until the slit length appears to be equal to the gap between the corneal endothelium and the anterior lens capsule as observed in the optical section. The method was applied to 56 eyes of 28 consecutive patients and compared with results obtained with the Haag-Streit pachometer. The equation deduced for predicting anterior chamber depth was

$$
x=\frac{(y+0 \cdot 21)}{0 \cdot 78}
$$

where $y=$ slit length and $x$ is the anterior chamber depth according to the pachometer measurement. The correlation between the two sets of results was 0.98 . The above relationship was converted to an approximate clinical relationship of anterior chamber depth = slit length $\times 1.4$ and this was said to be accurate to $95 \%$ confidence limits over the range of chamber depths encountered. It was claimed that a depth estimate accuracy of $\pm 0.2 \mathrm{~mm}$ relative to standard pachometry could be achieved.

Smith $^{5}$ also suggested a method of anterior chamber depth measurement, again using the HaagStreit 900 slit-lamp and its calibrated adjustable slit length facility. The technique utilises a horizontal slit with the angle between the observation and illumination system set at $60^{\circ}$. The patient is instructed to fixate the biomicroscope with the illumination system in the patient's temporal field, and the slit is focused 


\section{a. IMAGES NOT TOUCHING - slit length too short}

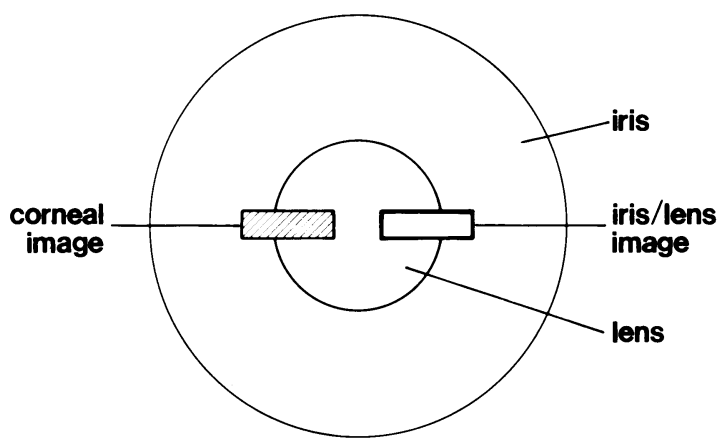

b. IMAGES JUST TOUCHING - slit length correct

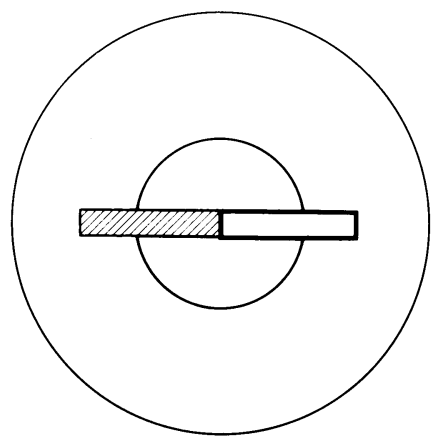

Fig. 1 Observer's view of slit-lamp images on cornea and iris/lens when using the method suggested by Smith. ${ }^{5}$

on the patient's cornea. The observer's view is illustrated in Fig. 1. The optical system is illustrated (in simplified form) in Fig. 2. As the slit length is increased, the gap between the cornea and lenticular slits decreases until the corneal slit appears to be just touching the lenticular slit. Smith recorded the slit length when this end point was reached and compared the result with the pachometric measurement of anterior chamber depth. Once again a high correlation of 0.966 was noted between slit length and pachometric depth measurement. The linear relationship between the two was deduced as $y=1 \cdot 117$, $x+0 \cdot 5079$, where $x=$ slit length and $y$ is the pachometric depth measurement. The correspondence subsequent to the publication of Smith's paper revealed an earlier attempt at anterior chamber depth measurement by Gorbani ${ }^{6}$ using a similar approach to the problem. The previous techniques provide for anterior chamber depth estimation without the need for special accessories. However, they require a slit-lamp with a calibrated variable slit

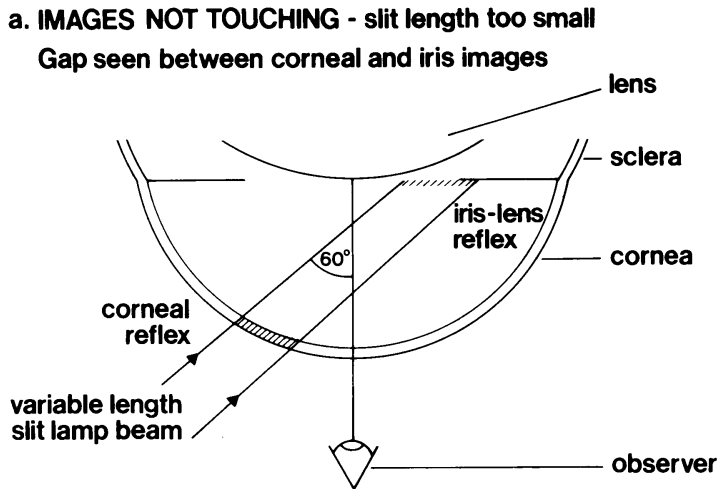

\section{b. IMAGES JUST TOUCHING - slit length correct}

Corneal images and iris image seen to touch

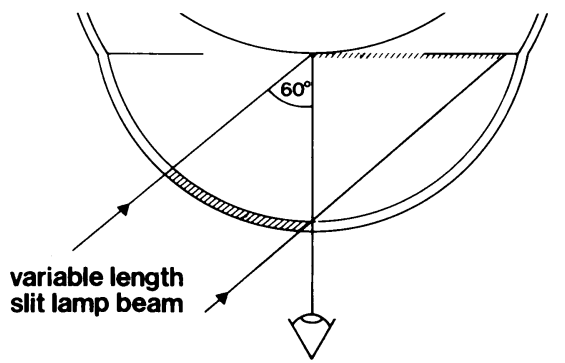

Fig. 2 Simplified optical diagrams of the slit beam traversing the anterior chamber in Smith's method.

length. It was believed that a modification of Smith's technique could provide a method for use with any slit-lamp.

\section{Method}

It was decided to follow the example of Jacobs ${ }^{4}$ and Smith $^{5}$ and measure the anterior chamber depth with the Haag-Streit pachometer to obtain a reliable indication of chamber depth in each subject. The pachometer was used following the recommendations of Lowe ${ }^{7}$ with two exceptions. Firstly, six measurements of chamber depth were taken and averaged. Secondly, a small piece of frosted Perspex was placed above the slit diaphragm. The patient was instructed to fixate the slit aperture so that the light fell on the corneal apex. The reflected light will produce an out-of-focus slit image on the Perspex screen, and this was aligned with the slit diaphragm by lateral adjustment of the slit-lamp position. This ensures that the same central section of the anterior eye was observed when the measurement was repeated.

The arrangement originally utilised for anterior 
chamber depth measurement without the pachometer was similar to that illustrated in Fig. 2 and involved the subject fixating the biomicroscope with the slit focused on to the cornea at an illumination system angle of about $80^{\circ}$ to the observation system. However, considerable difficulty was encountered in making observations, mainly owing to corneal reflections. The difficulties were minimised by interchanging the observation and illumination systems.

The method finally adopted was as follows. A horizontal slit of $2 \mathrm{~mm}$ length (obtained by using the aperture diaphragm disc) and approximately $0.5 \mathrm{~mm}$ width was projected on to the eye with the subject fixating the slit diaphragm. This small bright target is an aid to steady fixation, and the fixed position of the illumination system ensures that a depth measurement of the central region including the corneal apex is made in every subject independent of anterior chamber depth. The slit was centred in the pupillary aperture and was then adjusted until the corneal and lenticular slits appeared to have the same vertical dimension. Thus both images were slightly out of focus, with the true focus of the slit lying between the posterior cornea and anterior lens. The objective magnification was set at $1 \times$ with a $10 \times$ eyepiece. The observer viewed the anterior eye using the biomicroscope eyepiece nearest the illumination system at an angle of about $80^{\circ}$ on the temporal side. The observer's view at this point is similar to that illustrated in Fig. 1a. The angle between the observation and illumination systems was then reduced by moving the microscope until the temporal edge of the corneal slit appeared to be just touching the nasal edge of the lenticular slit as in Fig. 1b. The angle between the two systems was noted by means of the protractor scale on the slit-lamp main column. The angle can be estimated to the nearest $0.5^{\circ}$ if the Vernier scale is used. Six measurements were made on each eye and a mean calculated.

If it is assumed that the corneal and lenticular slits are formed in a plane and are separated by an anterior chamber depth $d$ of refractive index 1.336 , then

$$
d=\frac{2}{\tan \left\{\sin ^{-1}\left(\frac{\sin \theta}{1.336}\right)\right\}}
$$

where $\theta=$ angle between the observation and illumination systems. This is perhaps an oversimplification and may lead to some inaccuracy.

\section{Results}

Fifty eyes were examined in subjects with ages ranging from 19 to 57 years; six pachometric and six angular measurements were taken on each eye. The standard deviation of each set of six results was deduced in order to assess the likely variation in measurement. The mean standard deviation for the 50 eyes was $0.03 \mathrm{~mm}$ by pachometry and $0.04 \mathrm{~mm}$ by angular measurement. The mean and median depth for the 50 eyes were very similar, suggesting that the group is a normally distributed sample. The equation illustrated at the end of the method was used to deduce the anterior chamber depth. This correlated very well with the chamber depth measured by pachometer. Since the correlation coefficient was very high $(r=0.95)$, a linear regression equation was deduced to compare the depths predicted by both methods. The angular measurement gives a depth estimation which is $0.3 \mathrm{~mm}$ less than that indicated by the pachometer. This illustrates the inaccuracy arising from the equation used to deduce anterior chamber depth, which it will be recalled makes certain assumptions to simplify the mathematical treatment. A correction factor of $0.3 \mathrm{~mm}$ could be added to the depth calculated in order to give a more realistic result.

In clinical work it would be more convenient to use a simple table that converts the angular measurement into an anterior chamber depth. This can be derived

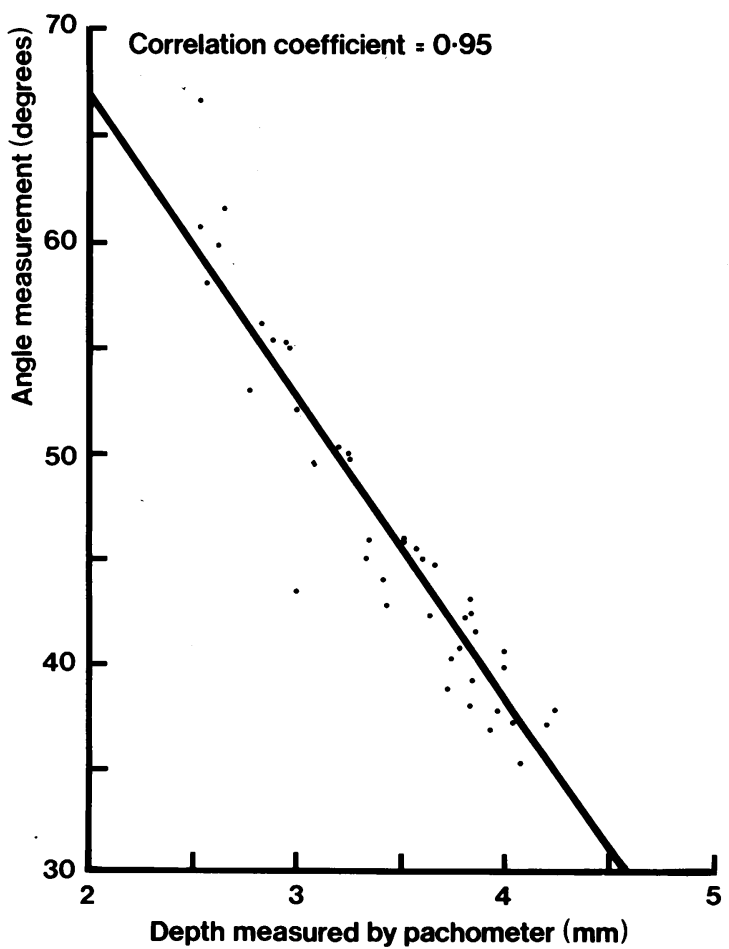

Fig. 3 Graph of anterior chamber depth measured by pachometer compared with the angular measurement between the observation and illumination systems of the slit-lamp following a modification of Smith's method. 
Table 1 Angle to anterior chamber depth conversion. This table was derived from the regression line illustrated in Fig. 3

\begin{tabular}{|c|c|c|c|}
\hline Angle ${ }^{\circ}$ & Depth, mm & Angle $^{\circ}$ & Depth, mm \\
\hline 35 & $4 \cdot 22$ & 49 & $3 \cdot 24$ \\
\hline 36 & $4 \cdot 15$ & 50 & $3 \cdot 17$ \\
\hline 37 & 4.08 & 51 & $3 \cdot 11$ \\
\hline 38 & 4.01 & 52 & 3.04 \\
\hline 39 & 3.94 & 53 & 2.97 \\
\hline 40 & 3.87 & 54 & 2.90 \\
\hline 41 & $3 \cdot 80$ & 55 & $2 \cdot 83$ \\
\hline 42 & 3.73 & 56 & $2 \cdot 76$ \\
\hline 43 & 3.66 & 57 & $2 \cdot 69$ \\
\hline 44 & 3.59 & 58 & $2 \cdot 62$ \\
\hline 45 & 3.52 & 59 & 2.55 \\
\hline 46 & 3.45 & 60 & 2.48 \\
\hline 47 & $3 \cdot 38$ & 61 & $2 \cdot 40$ \\
\hline 48 & $3 \cdot 31$ & 62 & $2 \cdot 34$ \\
\hline
\end{tabular}

following the example of Jacobs ${ }^{4}$ and $\mathrm{Smith}^{5}$ by analysing the angular measurement and the pachometry depth that accompanies it. Fig. 3 illustrates the relationship. The points on the graph mark the measurements taken. The relationship appears to be approximately linear over the range of chamber depths encountered in this work. The line on the graph is the linear regression line obtained by the least squares method and is of the form $y=14 \cdot 35 x+95 \cdot 56$, where $y$ is the angle between the observation and illumination systems and $x$ is the chamber depth indicated by the pachometer. This regression line is deduced on the assumption that the pachometer depth measurement is less likely to suffer from errors than the angular measurement. Once again the correlation coefficient is high at 0.95 , and so this regression can be used to deduce a table comparing angular measurement with expected chamber depth. Table 1 illustrates the relationship, which indicates that a 1 degree change of angle is required for a $0.07 \mathrm{~mm}$ change in depth over an angular range from $35^{\circ}$ to $62^{\circ}$ (depth range 4.22 to $2.34 \mathrm{~mm}$ ). Since the geometry of the system indicates that the relationship is not strictly linear, absolute depth predictions outside the range of values encountered in this study must be viewed with caution until the range is extended by examining more subjects, particularly with shallow anterior chambers. However, ray tracing by accurate calculation using the constants of Gullstrand's exact eye suggests that, for an anterior chamber depth of $1.5 \mathrm{~mm}$, the error induced by extrapolation of Table 1 results in an overestimation of chamber depth by $0.05 \mathrm{~mm}$. The calculations also suggest that total internal reflections will occur at the anterior corneal surface for a chamber depth around $1 \mathrm{~mm}$, which sets the lower limit for the technique. It may be appropriate to round the chamber depth values in Table 1 to the first decimal place. This approximation suggests that a $0 \cdot 1 \mathrm{~mm}$ change in chamber depth will be accompanied by a $1^{\circ}$ change in the angle between the observation and illumination systems. This approximation is not only easily remembered but compares favourably with the approximations of Jacobs ${ }^{4}$ and Smith.

\section{References}

1 Lindstedt F. Über die messung der Tiefe der vorderen Augenkammer mittels eines neuen, für klinischen Gebrauch bestimmten Instruments. Arch Augenheilkd 1916; 80: 104-67

2 Stenstrom S. An apparatus for the measurement of the depth of the anterior chamber based on the principle of Lindstedt. Acta Ophthalmol (Kbh) 1953; 31: 265-70.

3 Jaeger W. Tiegenmessung der menschlichen Vorderkammer mit plan parallelen plattern (Zusatzgerat zur Spaltlampe). Klin Exp Ophthalmol 1952; 153: 120-31.

4 Jacobs IG. Anterior chamber depth measurement using the slit lamp microscope. Am J Ophthalmol 1979; 88: 236-8.

5 Smith RJH. A new method of estimating the depth of the anterior chamber. Br J Ophthalmol 1979; 63: 215-20.

6 Gorbani A. Optico-geometric technic of determining the depth of the anterior chamber by means of a slit lamp (in Russian trans. from English summary) Vestn Oftalmol 1968; 47: 77-80.

7 Lowe RF. New instruments for measuring anterior chamber depth and corneal thickness. Am J Ophthalmol 1966; 62: 7-11.

Accepted for publication 2 July 1985. 